\title{
Patient-prosthesis mismatch after mitral valve-in-valve procedure - at the cost of life or serious consequence?
}

\author{
Dariusz Jagielak ${ }^{1}$, Radoslaw Kozaryn $^{1}$, Miłosz Jaguszewski², Jan Rogowski ${ }^{1}$ \\ ${ }^{1}$ Department of Cardiovascular Surgery, Medical University of Gdansk, Gdansk, Poland \\ ${ }^{2}$ Division of Cardiology, University Heart Center, University Hospital Zurich, Zurich, Switzerland
}

Postep Kardiol Inter 2015; 11, 2 (40): 154-155

DOI: $10.5114 /$ pwki.2015.52292

An 81-year-old man, who had undergone a complex cardiosurgical procedure 8 years previously (aortic valve replacement by Medtronic Hancock II $23 \mathrm{~mm}$ and mitral valve replacement by Medtronic Hancock II $31 \mathrm{~mm}$, tricuspid valve repair using the Kay technique, and coronary artery bypass surgery with left internal mammary artery (LIMA) to left anterior descending (LAD)), was admitted to the hospital with symptoms suggestive of acute heart failure (NYHA IV). Echocardiographic examination revealed satisfactory function of the aortic prosthesis, and massive regurgitation of the degenerative mitral prosthesis. Left ventricle ejection fraction was estimated at approximately $30 \%$ with left ventricle (LV) thinning, an akinetic and thin apex, and mild tricuspid valve insufficiency. Due to these findings cardiopulmonary bypass was used. An intra-aortic balloon pump (IABP) was not introduced due to limited femoral access. The patient, in spite of a very high operative risk (STS 29.8\%), was referred for urgent redo cardiac surgery. With this in mind, the patient was qualified for transcatheter mitral valvein-valve (VIV) implantation.

The procedure was performed on-pump with the cardiopulmonary bypass connected via femoral vessels. A transapical approach for delivering the $29 \mathrm{~mm}$ Edwards Sapien valve into the mitral position was chosen. Significant efforts were made to protect the scarred apex (pericardium was not dissected). Intraoperative transesophageal echocardiogram (TEE) and fluoroscopy demonstrated precise landing of the new prosthesis, as well as resolution of prior regurgitation and absence of perivalvular leakage. Unfortunately, the initially high mean gradient through the valve remained elevated. The postoperative course was uneventful. On postoperative day 5 in the Cardiology Department due to left ventricular end-diastolic diameter (LVED) > $70 \mathrm{~mm}$ and one incidence of tachyarrhythmia, a cardioverter-defibrillator was implanted prophylactically. The patient was discharged home 2 weeks after the procedure. The patient remains in stable condition in class II/II NYHA after 10 months of follow-up. Control echocardiography showed moderate/severe stenosis (mitral valve area $1.0 \mathrm{~cm}^{2}$, mean diastolic pressure gradient (PG) $9 \mathrm{~mm}$ $\mathrm{Hg}$ ) of the implanted VIV prosthesis with no perivalvular leakage and degenerative changes of the leaflets.

Though the problem of low mitral valve orifice after transcatheter VIV implantation has been deliberated in the literature, in our opinion it has not been outlined in sufficient detail [1]. It is not entirely clear if the patient-prosthesis mismatch (PPM) in our case was influenced by the anatomy of this particular patient, or is rather caused by the atypical position of the bioprosthesis, which was originally designed for the aortic position [2]. From a technical point of view the procedure was executed correctly with maximum balloon inflation during implantation and precise prosthesis positioning. The range of mean diastolic pressure gradients through the mitral orifice after VIV procedures is expansive in available data. We presume, according to our experience, that the rigidity of the biological tissue of which the leaflets are made does not allow complete opening under the diastolic pressure gradients between the left atrium (LA) and LV, which are physiologically much lower than that of the aortic valve [3]. Although VIV has been performed approximately a few hundred times worldwide, there are still quite limited data regarding follow-up of these patients after this procedure [4].

Corresponding author:

Dr Radoslaw Kozaryn, Department of Cardiovascular Surgery, Medical University of Gdansk, 7 Dębinki St, 80-952 Gdansk, Poland, phone: +48 5834924 03, fax: +48 5834176 69, e-mail: rkozaryn@uck.gda.pl

Received: 9.01.2015, accepted: 29.03.2015. 

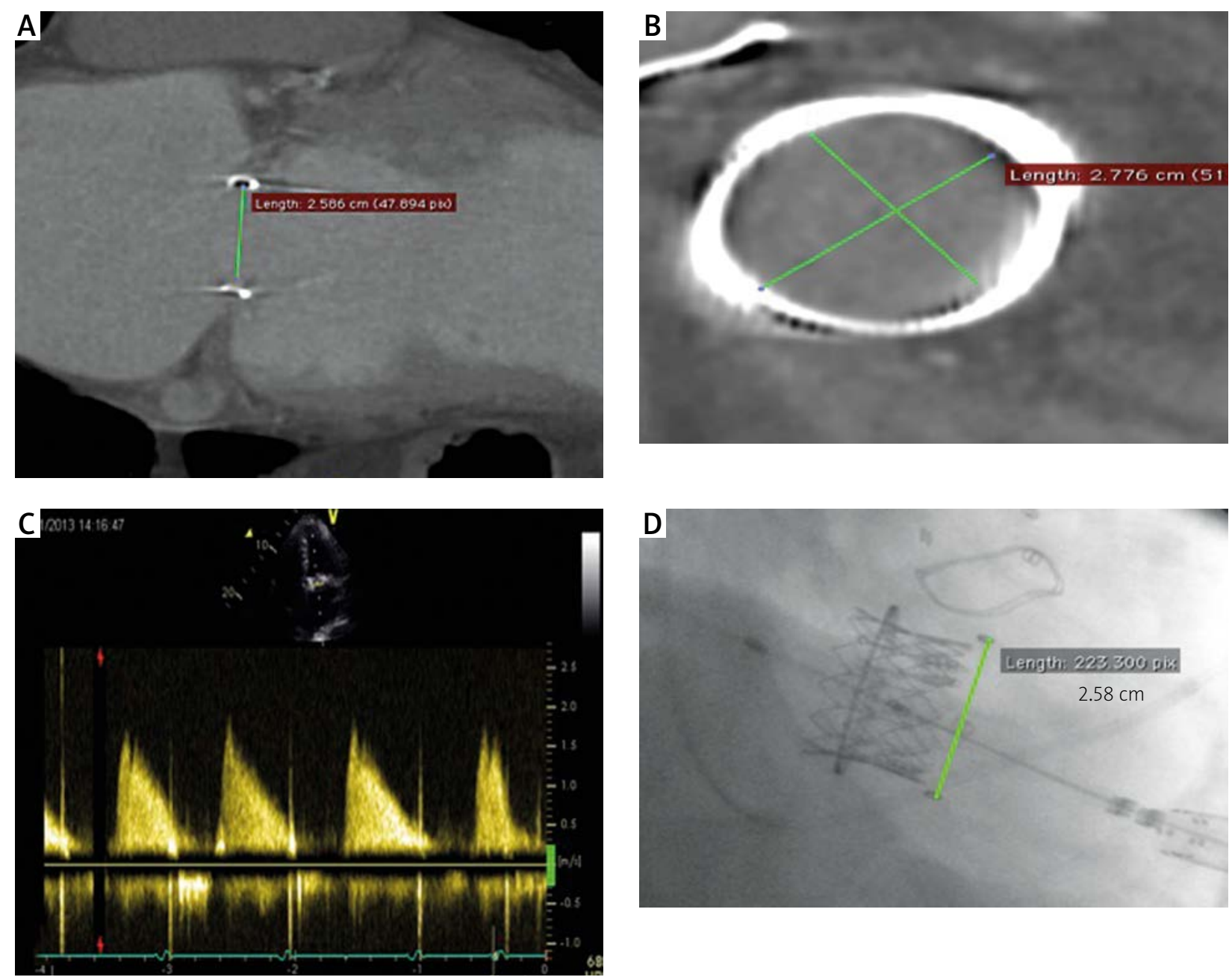

Figure 1. A, B - Angio-CT - internal diameter sizing of the bioprosthesis annulus $(\mathbf{A}-2.58 \mathrm{~cm}, \mathbf{B}-2.78 \mathrm{~cm})$. C - Doppler showing no regurgitation, but the orifice area is still not optimal. D - Angiography after implantation

\section{Conflict of interest}

The authors declare no conflict of interest.

\section{References}

1. Bapat V, Adams B, Attia R, et al. Neo-annulus: a reference plane in a surgical heart valve to facilitate a valve-in-valve procedure. Catheter Cardiovasc Interv 2015; 85: 685-91.

2. Bapat V, Attia R, Redwood S, et al. Use of transcatheter heart valves for a valve-in-valve implantation in patients with degenerated aortic bioprosthesis: technical considerations and results. J Thorac Cardiovasc Surg 2012; 144: 1372-9.

3. Noorani A, Attia R, Bapat V, et al. Valve-in-valve procedure: importance of the anatomy of surgical bioprostheses. Multimed Man Cardiothorac Surg 2014 Oct 29; 2014 pii: mmu020. doi: 10.1093/ mmcts/mmu020.

4. Raval J, Nagaraja V, Eslick GD, Denniss AR. Transcatheter valve-invalve implantation: a systematic review of literature. Heart Lung Circ 2014; 23: 1020-8. 